\title{
Tolerância de genótipos de cereais de inverno ao alumínio em cultivo hidropônico e em campo
}

\author{
Roberto Portaluppi ${ }^{(1)}$, Sandra Patussi Brammer(2), Jurandir Vieira de Magalhães(3), Cibele Tesser da Costa(2), \\ Eduardo Caierão ${ }^{(2)}$, Alfredo do Nascimento Junior ${ }^{(2)}$ e José Pereira da Silva Junior ${ }^{(2)}$
}

(1)Universidade de Passo Fundo, Faculdade de Agronomia e Medicina Veterinária, Rodovia BR 285, Km 291, CEP $99001-970$ Passo Fundo, RS. E-mail: roberto.luppi@bol.com.br (2)Embrapa Trigo, Rodovia BR 285, Km 294, CEP 99001-970 Passo Fundo, RS. E-mail: sandra@cnpt.embrapa.br, cibeletc@yahoo.com.br, caierao@cnpt.embrapa.br, alfredo@cnpt.embrapa.br, jpereira@cnpt.embrapa.br ${ }^{(3)}$ Embrapa Milho e Sorgo, Rodovia MG 424, Km 45, CEP 35701-970 Sete Lagoas, MG. E-mail: jurandir@cnpms.embrapa.br

Resumo - O objetivo deste trabalho foi determinar a capacidade de crescimento radicular de 75 genótipos de cereais de inverno em cultivo hidropônico, em diferentes concentrações de alumínio, avaliar a relação entre o grau de tolerância/sensibilidade, em solução hidropônica, e a resistência/suscetibilidade ao crestamento em campo. Os cereais cevada, triticale, centeio, trigo e Aegilops tauschii foram avaliados em hidroponia, com concentrações de $\mathrm{Al}^{3+}$ que variaram entre 0,5 (cevada), 2 e 6 (triticale), 6 e 10 (centeio) e $2 \mathrm{mg} \mathrm{L}^{-1}$ (trigo e Ae. tauschii). Os delineamentos experimentais foram inteiramente casualizados. Em campo, foram avaliados os mesmos genótipos, exceto Ae. tauschii, em solo com pH 4,4 e 4,85, corrigido a 1/2 e 1/4 do índice SMP. Utilizou-se uma escala de notas com variação de escores de 0,5 (altamente resistente) a 5 (altamente suscetível). Foi observada elevada relação entre a tolerância ao alumínio em hidroponia e a resistência ao crestamento em campo. A seleção de cereais em meio hidropônico pode ser considerada eficiente como ferramenta de apoio aos programas de melhoramento genético para essa característica.

Termos para indexação: Hordeum vulgare, Secale cereale, Triticum aestivum, crestamento, resistência, suscetibilidade, toxicidade.

\section{Tolerance of small graine cereal genotypes to aluminum on hydroponic and field cultivation}

\begin{abstract}
The objective of this work was to determine the root growth capacity of 75 genotypes of small graine cereals in hydroponic culture under different aluminum concentrations, and to assess the relationship betwen the level of tolerance/sensitivity in hydroponic solution and the resistance/susceptibility index in the field. Barley, triticale, rye, wheat and Aegilops tauschii were evaluated in hydroponics, with $\mathrm{Al}^{3+}$ concentrations that varied between 0.5 (barley), 2 and 6 (triticale), 6 and 10 (rye) and $2 \mathrm{mg} \mathrm{L}^{-1}$ (wheat and Ae. tauschii). The experiments' designs were completely randomized. In the field trial, the same genotypes were assessed, except for Ae. tauschii, in soil with $\mathrm{pH} 4.4$ and 4.85 adjusted to $1 / 2$ and $1 / 4$ of the SMP index. A score scale ranging from 0.5 (highly resistant) to 5 (highly susceptible) was used. A strong relationship between aluminum tolerance in hydroponics and resistance to blight in the field was observed. Cereals selection in hydroponic medium can be considered an efficient tool to support breeding programs for this characteristic.
\end{abstract}

Index terms: Hordeum vulgare, Secale cereale, Triticum aestivum, blight, resistance, sensitivity, toxicity.

\section{Introdução}

A toxicidade do alumínio, nos solos ácidos, é um dos fatores que mais limitam a produção agropecuária. No Brasil, 68\% dos solos têm elevada acidez, toxicidade de alumínio e baixa disponibilidade de fósforo (Guimarães, 2005). Cultivares de cereais de inverno suscetíveis ao $\mathrm{Al}^{3+}$ apresentam, como sintomas, raízes atrofiadas, folhas amarelecidas, formação de espigas rudimentares, com poucas ou até mesmo sem espiguetas, o que é conhecido como crestamento.
A combinação de métodos de análise como o cultivo hidropônico e o cultivo em campo pode ser essencial para identificar genótipos que têm múltiplos mecanismos de tolerância a essa característica, e permite avaliar a redução do crescimento radicular dos cereais de inverno, provocada pelo alumínio tóxico na forma do cátion $\mathrm{Al}^{3+}$ (Narasimhamoorthy et al., 2007). O cultivo hidropônico permite a medição das raízes de modo mais preciso que o cultivo em solo, pois as estruturas sofrem menos danos físicos ao 
serem retiradas da solução nutritiva. A hidroponia é um método de caracterização específica da toxicidade do alumínio, enquanto o solo apresenta outros fatores potenciais ligados à toxicidade, como os teores elevados de ferro e manganês e a deficiência de fósforo e de molibdênio. Em experimentos em campo, o pH e os teores dos elementos químicos do solo variam constantemente conforme as variações climáticas, e a toxicidade ao alumínio não é o único fator limitante. Além disso, as raízes, que são a parte mais afetada pelo alumínio, não são facilmente observadas. Nos métodos hidropônicos, essas variáveis e também os estudos genéticos e a caracterização de germoplasma específico para a tolerância ao alumínio são passíveis de serem controladas (Camargo et al., 2006; Voss et al., 2006).

O objetivo do deste trabalho foi determinar a capacidade de crescimento radicular de genótipos de cevada, triticale, centeio, trigo e Aegilops tauschii cultivados em hidroponia, em diferentes concentrações de alumínio, e avaliar a relação entre a tolerância/ sensibilidade dos genótipos em solução hidropônica com a resistência/suscetibilidade ao crestamento em campo.

\section{Material e Métodos}

Os experimentos foram realizados no Laboratório de Biotecnologia e no campo experimental da Embrapa Trigo, localizado em Passo Fundo, RS, a $28^{\circ} 15^{\prime} \mathrm{S}$, $52^{\circ} 24^{\prime} \mathrm{W}$ e a $687 \mathrm{~m}$ de altitude. O solo do local do experimento de campo é classificado como Latossolo Vermelho distrófico típico segundo Streck (2002). Foram avaliados 75 genótipos, tendo sido 14 de cevada (Hordeum vulgare L.); 15 de triticale (X Triticosecale Wittmack); 15 de centeio (Secale cereale L.); 16 de trigo (Triticum aestivum L.) e 15 de Aegilops tauschii (=Triticum tauschii), mantidos pelo Banco Ativo de Germoplasma (BAG) da Embrapa Trigo (Tabela 1). Em todos os experimentos foram usadas as cultivares de trigo Anahuac 75 (sensível ao alumínio) e IAC 5-Maringá (tolerante ao alumínio), como controles para o caráter tolerante e sensível ao íon $\mathrm{Al}^{3+}$. Para o triticale, utilizaram-se as cultivares IAPAR 23-Arapoti (tolerante) e PFT 209 (sensível) como testemunhas, além dos controles de trigo. Quanto ao centeio, os genótipos BRS Serrano e 698210, reconhecidos como tolerante e moderadamente sensível, respectivamente, foram utilizados como testemunhas. Para o trigo, foram
Tabela 1. Genótipos de cevada, triticale, centeio e trigo do Banco Ativo de Germoplasma da Embrapa Trigo e seus respectivos cruzamentos utilizados para a realização dos experimentos.

\begin{tabular}{|c|c|}
\hline Genótipo & Cruzamento \\
\hline \multicolumn{2}{|l|}{ Cevada } \\
\hline Antártica 04 & Seleção de W-6601 \\
\hline PFC 7802 & Binder*2/Opal //Balder/Kenia/3/Alpha \\
\hline Cevada BR 1 & Binder/Opal//Balder/Kenia \\
\hline Harrington & Klages//Gazelle/Betzes \\
\hline IPFC 20011 & Seleção de Scarlett \\
\hline Alpha & Manchuria/Champion of Vermont \\
\hline BRS 224 & Embrapa 43/PFC 9114 \\
\hline FM 404 & Seleção de Alpha \\
\hline Antártica 01 & Seleção de Volla \\
\hline Antártica 05 & Origem desconhecida \\
\hline MN 698 & MN 599/MN 635 \\
\hline Cevada BR 1 & Binder/Opal//Balder/Kenia \\
\hline Cevada BR 2 & FM 424/Norbert \\
\hline BRS Marciana & PFC 9240/PFC 9211 \\
\hline FM 519 & Km1/Union//Volla/3/Km2/Volla//Wisa/4/Alpha \\
\hline \multicolumn{2}{|r|}{ 年 } \\
\hline Triticale BR1 & $\mathrm{M} 2 \mathrm{~A} / \mathrm{CML}$ \\
\hline Embrapa 53 & LT1117.62/Civet/Tatu \\
\hline PFT 112 & PFT 512/Guará \\
\hline IPR 111 & Anoas 5/Stier 13 \\
\hline BRS 148 & Yogui/Tatu \\
\hline BRS Ulisses & Erizo/Nimir \\
\hline BRS 203 & LT-1/Rhino \\
\hline BRS Netuno & Pollmer//2*Erizo/Bull-1 \\
\hline PFT 209 & PFT215*2/TCA 3050-89 \\
\hline IAPAR 23Arapoti & $\mathrm{CIN} / \mathrm{CNO} / / \mathrm{BGL} / 3 /$ Merino \\
\hline PFT 0407 & ERIZ011*2/Milman*2/Picus \\
\hline PFT 0609 & Embrapa 53//PFT 116/HOH-87.102-6-1 \\
\hline PFT 307 & PFT 312/PFT 511 \\
\hline BRS Minotauro & Octo 92-3(PF9358/CBR1TriticaleBR4 \\
\hline PFT 0417 & Presto//2*Tesmo-1 Mus 603/4/Ardi-1/... \\
\hline \multicolumn{2}{|l|}{ Centeio } \\
\hline PFS 0604 & Pop. Cent. 981 \\
\hline PFS 0603 & Pop. Cent. Desc. \\
\hline PFS 0501 & Pop. 8011 \\
\hline Centeio BR 1 & Seleção de pop.coloniais brasileiras \\
\hline IPR 89 & Seleção de pop. Australianas \\
\hline BRS Serrano & Centeio Garcia/Centeio Bagé \\
\hline 698210 & Pl. Rfco. no $\mathrm{Al}+03(\mathrm{Cd})$ \\
\hline PFS 0504 & Pop. Estefano Schneider \\
\hline 698206 & Pl. Rfco. no $\mathrm{Al}+03$ (Cd) \\
\hline 698207 & Pl. Rfco. no $\mathrm{Al}+03$ (Cd) \\
\hline PFS 0607 & Sel. Pl. Bx. Color escura \\
\hline PFS 0606 & Sel. Pl. Bx. Color clara \\
\hline PFS 0605 & Sel. Pl. Sel Al ${ }^{3+}+$ Rfco \\
\hline PFS 0502 & Pop. Andreas Kreuscha \\
\hline PFS 0601 & Sel. 3pl. \\
\hline \multicolumn{2}{|l|}{ Trigo } \\
\hline BRS Guamirim & Embrapa 27/Buck Nandu//PF 93159 \\
\hline BRS 194 & (2000) CEP14/BR23//CEP17 \\
\hline Jesuíta & Polyssu/ Alfredo Chaves 3-21 \\
\hline CD 104 & PFAU SIB/ Iapar 17 \\
\hline IPR 85 & Iapar 30/BR 18 \\
\hline PF 844005 & $\begin{array}{l}\text { T. durum Hércules,NE 22912/Ae. squarrosa, } \\
\text { NE } 20211 \text {-C }\end{array}$ \\
\hline Anahuac 75 & II 12300//Lerma Rojo64/8156/3/Norteno 67 \\
\hline IAC 5-Maringá & Frontana/ Kenya 54//PG1 \\
\hline BRS Buriti & Embrapa 27/Klein Orion \\
\hline IPR 87 & IOC 878/ Iapar 17 \\
\hline BRS Angico & PF $87107 / 2 *$ IAC 13 \\
\hline BRS 229 & Embrapa $27 * 3 / /$ BR35/Buck Poncho \\
\hline BRS Guabiju & PF $86743 /$ BR 23 \\
\hline BRS 209 & Iupateco 73/Embrapa 16 \\
\hline BRS Tarumã & Century/BR 35 \\
\hline
\end{tabular}


utilizadas as cultivares IAC 5-Maringá (tolerante) e Anahuac 75 (sensível), como controles e testemunhas do experimento. Além dos testes com os genótipos de trigo, para identificar novas fontes de tolerância, o mesmo estudo foi feito com a espécie Ae. tauschii e, do mesmo modo que o trigo, foram utilizadas as cultivares de trigo IAC 5-Maringá (tolerante) e Anahuac 75 (sensível) como controle e como testemunha.

As sementes foram previamente desinfestadas com hipoclorito de sódio a $2 \%$, por 4 min, seguida de seis enxágues com água destilada e deionizada. Após, foram semeadas em papel germitest e mantidas em câmara de crescimento com temperatura de $4^{\circ} \mathrm{C}$, por 24 horas, seguido por um período de 36 a 40 horas, com temperatura de $19-21^{\circ} \mathrm{C}$. Para os genótipos de $A e$. tauschii, as sementes foram mantidas, primeiramente, em temperatura de $2^{\circ} \mathrm{C}$, por 26 dias, a fim de promover a quebra de dormência. De 15 a 30 sementes germinadas, foram selecionadas oito de cada genótipo, com tamanho similar e com raízes de $0,5 \mathrm{~cm}$ de comprimento, que foram transferidas para uma tela de plástico com dois milímetros de malha, onde foram mantidas em flutuação, dentro de bandejas de plástico. As raízes foram inseridas nos orifícios da tela, acrescentando-se $2.000 \mathrm{~mL}$ de solução nutritiva.

Inicialmente foram preparadas soluções estoque individualizadas com os reagentes químicos P.A.: $\mathrm{CaCl}_{2} \cdot 2 \mathrm{H}_{2} \mathrm{O}, 58,80 \mathrm{~g} \mathrm{~L}^{-1} ; \mathrm{KNO}_{3}, 65,70 \mathrm{~g} \mathrm{~L}^{-1}$; $\mathrm{MgCl}_{2} \cdot 6 \mathrm{H}_{2} \mathrm{O}$, 50,80 g L L $\left(\mathrm{NH}_{4}\right)_{2} \mathrm{SO}_{4}, 1,30 \mathrm{~g} \mathrm{~L}^{-1}$; $\mathrm{NH}_{4} \mathrm{NO}_{3}, 3,20 \mathrm{~g} \mathrm{~L}^{-1}$. A solução nutritiva foi preparada com a concentração e composição de $400 \mu \mathrm{mol} \mathrm{L}^{-1}$ de $\mathrm{CaCl}_{2} ; 650 \mu \mathrm{mol} \mathrm{L}-1$ de $\mathrm{KNO}_{3} ; 250 \mu \mathrm{mol} \mathrm{L}^{-1}$ de $\mathrm{MgCl}_{2}$; $10 \mu \mathrm{mol} \mathrm{L}^{-1}$ de $\left(\mathrm{NH}_{4}\right)_{2} \mathrm{SO}_{4} ; 40 \mu \mathrm{mol} \mathrm{L}^{-1}$ de $\mathrm{NH}_{4} \mathrm{NO}_{3}$ (pH 4,0). Foi usado $1 \mathrm{~mL}$ da solução estoque de cada sal por litro de água destilada e deionizada. $\mathrm{O} \mathrm{pH}$ foi corrigido para 4, com o uso de $\mathrm{HCl}$. A solução estoque de alumínio foi preparada dissolvendo-se 0,894 $\mathrm{g}$ de $\mathrm{AlCl}_{3}$ com água destilada, com concentração final de $1 \mathrm{mg} \mathrm{mL}{ }^{-1}$ de Al.

O método para a avaliação de tolerância ao alumínio em hidroponia, em todos os cereais de inverno, foi baseado em Voss et al. (2006), com adaptações. As concentrações $\left(\mathrm{mg} \mathrm{L}^{-1} \mathrm{de} \mathrm{Al}^{3+}\right.$ ) de alumínio solúvel foram alteradas conforme a espécie: cevada, 0,5; triticale, 2 e 6; centeio, 6 e 10; trigo e Ae. tauschii, 2. Em todos os testes foi utilizada uma câmara de crescimento com controle de temperatura $\left(22-24^{\circ} \mathrm{C}\right)$ e de luz (24 horas). As soluções nas bandejas foram aeradas por meio de duas cânulas de vidro, instaladas em suas laterais e conectadas a um compressor de ar. Em uma das bandejas, foi adicionado alumínio, enquanto a outra permaneceu apenas com a solução nutritiva, que serviu como grupo controle. As soluções estoques de alumínio, específicas para cada espécie, foram substituídas em 24, 48 e 72 horas. Após 96 horas de crescimento, foram medidos os comprimentos da raiz principal e da raiz secundária maior, com paquímetro digital, modelo 727, (Starret, Ohio, USA). A medida do comprimento das duas raízes maiores é considerada a mais apropriada avaliação para estudos genéticos e moleculares e fornecem uma boa separação dos genótipos tolerantes e sensíveis ao alumínio (Wang et al., 2006).

Os experimentos foram instalados em delineamento inteiramente casualizado, em que cada planta foi considerada uma repetição. Foi utilizado o Procedimento GLM do SAS (SAS Institute, 2004). Para a análise estatística, considerou-se a média da medida das raízes de cada planta. A interpretação de tolerância seguiu o método de Voss et al. (2006). Inicialmente, as plantas foram retiradas do freezer e suas raízes foram mergulhadas em água, à temperatura ambiente, a fim de promover o descongelamento. Considerou-se sensível o material semelhante à testemunha sensível; e tolerante, o material semelhante à testemunha tolerante para cada espécie. Os genótipos com crescimento radicular intermediário foram considerados moderadamente sensíveis, quando apresentavam índices numéricos de 26 a 50\% maiores do que a média da testemunha sensível e moderadamente tolerante quando seus índices estavam entre 51 e $75 \%$ do valor obtido pela testemunha tolerante.

Afim de comparar a eficiência do método hidropônico aos resultados verificados nos ensaios em campo, os mesmos genótipos foram semeados no campo experimental da Embrapa Trigo. Os genótipos foram testados em área de solo caracterizado como Latossolo Vermelho distrófico típico, textura argilosa (Streck, 2002), concentrações de $\mathrm{pH}$ 4,4 e 4,85, disponíveis na mesma área experimental e que fazem parte de uma área conduzida para esse tipo de avaliação, que foi corrigida, respectivamente, a $1 / 2$ e $1 / 4$ do índice SMP. A cultivar suscetível ao crestamento, trigo Anahuac 75, serviu como controle dos experimentos.

A semeadura dos genótipos de cevada, de triticale, de centeio e de trigo foi feita em agosto de 2007, com 60 
sementes por metro linear, as quais foram previamente multiplicadas em 2006. As parcelas foram constituídas por cinco linhas de $1 \mathrm{~m}$ de comprimento com $20 \mathrm{~cm}$ de espaçamento entre as linhas, sem repetições. Para a espécie Ae. tauschii não foi feito cultivo e análise em campo, uma vez que essa espécie não apresenta tipo agronômico adequado para plantio. As doenças das plantas nas parcelas foram controladas pela aplicação dos fungicidas Piraclostrobina + Epoxiconazole, na dosagem de $1.333 \pm 50$ g i.a. ha ${ }^{-1}$, nos estádios de afilhamento e espigamento. $\mathrm{O}$ controle de plantas daninhas foi feito por meio de capina manual.

A avaliação do experimento no campo foi realizada em novembro de 2007, durante o ciclo vegetativo e seguiu o método de Sousa (1998), que classifica os genótipos com escala de notas de 0 a 5 em relação a tolerância/sensibilidade ao alumínio, em que: altamente resistente $(0,50-0,80)$; resistente $(0,81-1,50)$; moderadamente resistente $(1,51-$ $2,50)$, moderadamente suscetível $(2,51-3,50)$; suscetível $(3,51-4,50)$ e altamente suscetível $(4,51-5,0)$. O critério dos escores utilizado foi: 0,5 , altamente resistente; 1 , resistente, plantas normais e vigorosas com perfilho abundante; 2 , moderadamente resistente, plantas normais, levemente menos vigorosas e com menos perfilhos do que o grupo anterior; 3, moderadamente suscetíveis, desenvolvimento das plantas intermediário; 4 , suscetíveis, plantas deficientes, sem perfilho; e 5, altamente suscetíveis, plantas mortas ou com desenvolvimento muito deficiente.

A análise de variância para genótipo e concentração de Alumínio foram efetuadas pelo procedimento GLM do

Tabela 2. Classificação quanto à tolerância e à sensibilidade de genótipos de cevada ao $\mathrm{Al}^{3+}$, definida pelo comprimento da raiz de plantas em cultivo hidropônico em solução de $\mathrm{Al}^{3+}$, que tiveram como controle as cultivares de trigo Anahuac 75 (sensível) e IAC 5-Maringá (tolerante).

\begin{tabular}{lccc}
\hline Genótipo & \multicolumn{2}{c}{ Comprimento $(\mathrm{mm})$} & Classificação $^{(1)}$ \\
\cline { 2 - 3 } & $0^{(2)}$ & $0,5^{(2)}$ & \\
\hline Antártica 04 & 31,21 & 19,71 & MT \\
PFC 7802 & 29,86 & 26,93 & $\mathrm{~T}$ \\
Cevada BR 1 & 38,64 & 25,40 & MT \\
Harrington & 31,89 & 16,00 & MS \\
Anahuac 75 & 32,31 & 14,36 & $\mathrm{~S}$ \\
IAC 5-Maringá & 53,43 & 62,71 & $\mathrm{~T}$ \\
IPFC 20011 & 32,07 & 20,64 & MT \\
Alpha & 22,34 & 11,71 & MS \\
BRS 224 & 26,10 & 15,85 & MT \\
FM 404 & 21,21 & 16,33 & MT \\
Antártica 01 & 23,39 & 27,53 & $\mathrm{~T}$ \\
\hline
\end{tabular}

${ }^{(1)} \mathrm{T}$, tolerante; S, sensível; MT, moderadamente tolerante; MS, moderadamente sensível. ${ }^{(2)}$ Concentração de $\mathrm{Al}^{3+} \mathrm{em} \mathrm{mg} \mathrm{L}{ }^{-1}$.
FAS (SAS Institute 2004) e as diferenças determinadas pelo teste de Fukey a 5\% de probabilidade.

\section{Resultados e Discussão}

Dos 14 genótipos de cevada testados, as cultivares PFC 7802 e Antártica 01 foram incluídas no grupo dos genótipos tolerantes ao alumínio trocável (Tabela 2). As cultivares Harrington e Alpha foram incluídas no grupo dos moderadamente sensíveis. Os genótipos MN 698, Cevada BR2, BRS Marciana, FM 519 e Antártica 05 apresentaram baixo índice de germinação, o que inviabilizou a sua avaliação. As cultivares Harrington e Alpha não diferiram $(\mathrm{p}=0,05)$ da cultivar de trigo Anahuac 75 sensível ao alumínio, e 69,5\% da variação observada foi explicada com o modelo ajustado. Esses resultados podem ser explicados em razão de que a cevada é considerada o cereal mais sensível à toxicidade do alumínio. Sua tolerância ao alumínio é controlada por um loco simples, de um gene localizado no cromossomo $4 \mathrm{H}$, e sua base genética para a tolerância é muito pequena (Wang et al., 2006).

Quanto ao triticale, no grupo dos tolerantes, situaram-se as cultivares IAPAR 23-Arapoti, Embrapa 53, PFT 112, IPR 111, BRS 148, BRS Ulisses, PFT 0407, PFT 0609

Tabela 3. Classificação quanto à tolerância e à sensibilidade de genótipos de triticale ao $\mathrm{Al}^{3+}$, definida pelo comprimento da raiz de plantas em cultivo hidropônico em solução de $\mathrm{Al}^{3+}$, que tiveram como controle as cultivares de trigo Anahuac 75 (sensível) e IAC 5-Maringá (tolerante), e como testemunhas as cultivares de triticale PFT 209 (sensível) e IAPAR 23-Arapoti (tolerante).

\begin{tabular}{lccc}
\hline Genótipo & \multicolumn{2}{c}{ Comprimento $(\mathrm{mm})$} & Classificação $^{(1)}$ \\
\cline { 2 - 3 } & $0^{(2)}$ & $6^{(2)}$ & \\
\hline Triticale BR1 & 32,02 & 12,67 & $\mathrm{~S}$ \\
Embrapa 53 & 32,98 & 22,49 & $\mathrm{~T}$ \\
PFT 112 & 36,41 & 21,48 & $\mathrm{~T}$ \\
IPR 111 & 35,42 & 23,55 & $\mathrm{~T}$ \\
BRS 148 & 32,06 & 23,29 & $\mathrm{~T}$ \\
BRS Ulisses & 42,88 & 27,85 & $\mathrm{~T}$ \\
IAC 5-Maringá & 67,21 & 26,50 & $\mathrm{~T}$ \\
Anahuac 75 & 52,23 & 9,14 & $\mathrm{~S}$ \\
BRS 203 & 31,15 & 14,39 & $\mathrm{MT}$ \\
BRS Netuno & 35,88 & 16,27 & $\mathrm{MT}$ \\
PFT 209 & 39,47 & 13,94 & $\mathrm{~S}$ \\
IAPAR 23 -Arapoti & 33,89 & 23,04 & $\mathrm{~T}$ \\
PFT 0407 & 39,20 & 21,26 & $\mathrm{~T}$ \\
PFT 0609 & 34,84 & 19,04 & $\mathrm{~T}$ \\
PFT 307 & 39,02 & 17,75 & MT \\
BRS Minotauro & 32,99 & 24,33 & $\mathrm{~T}$ \\
\hline${ }^{(1)}$ T, tolerante; S, sensível; MT, moderadamente tolerante. ${ }^{(2)}$ Concentração \\
de Al ${ }^{3+}$ em mg L ${ }^{-1}$. & & &
\end{tabular}

Pesq. agropec. bras., Brasília, v.45, n.1, p.178-185, jan. 2010 
e BRS Minotauro, e no dos sensíveis, PFT 209 e Triticale BR1 (Tabela 3). A linhagem PFT 0417 apresentou baixo índice de germinação, o que impossibilitou a sua avaliação. Os genótipos tolerantes e moderadamente tolerantes não diferiram $(p=0,05)$ do IAPAR 23-Arapoti. Com o modelo estatístico ajustado, $79,5 \%$ da variação observada foi explicada.

O triticale é um híbrido de trigo e centeio, o qual apresenta diferentes níveis de tolerância ao $\mathrm{Al}^{3+}$. Essa tolerância, advinda principalmente do parental centeio, é muito importante para os programas de melhoramento dessa cultura (Aniol \& Gustafson, 1984). Provavelmente, seja esta a explicação dos resultados obtidos nesta avaliação.

Os genótipos de centeio PFS 0501, IPR 89, PFS 0502, PFS 0504, PFS 0605, PFS 0606, PFS 0607, 698206 e 698207 foram incluídos no grupo dos tolerantes e o PFS 0604, moderadamente sensível (Tabela 4). Para essa espécie, $88,9 \%$ da variação observada foi explicada com o modelo ajustado.

Em estudo comparativo realizado entre sete populações de centeio, cultivadas há vários séculos por agricultores do norte de Portugal e 11 cultivares

Tabela 4. Classificação quanto à tolerância e à sensibilidade de genótipos de centeio ao $\mathrm{Al}^{3+}$, definida pelo comprimento da raiz de plantas em cultivo hidropônico em solução de $\mathrm{Al}^{3+}$, que tiveram como controle as cultivares de trigo Anahuac 75 (sensível) e IAC 5-Maringá (tolerante) e, como testemunhas, as cultivares de centeio BRS Serrano (tolerante) e 698210 (sensível) .

\begin{tabular}{|c|c|c|c|}
\hline \multirow[t]{2}{*}{ Genótipo } & \multicolumn{2}{|c|}{ Comprimento (mm) } & \multirow[t]{2}{*}{ Classificação $^{(1)}$} \\
\hline & $0^{(2)}$ & $10^{(2)}$ & \\
\hline PFS 0604 & 59,99 & 15,63 & MS \\
\hline PFS 0603 & 64,96 & 23,53 & MT \\
\hline PFS 0501 & 63,24 & 29,40 & $\mathrm{~T}$ \\
\hline Centeio BR 1 & 68,81 & 26,33 & MT \\
\hline IPR 89 & 76,36 & 34,42 & $\mathrm{~T}$ \\
\hline Anahuac 75 & 34,12 & 4,96 & S \\
\hline IAC 5-Maringá & 64,74 & 16,89 & $\mathrm{~T}$ \\
\hline BRS Serrano & 61,75 & 30,79 & $\mathrm{~T}$ \\
\hline 698210 & 49,06 & 12,57 & MS \\
\hline PFS 0504 & 76,36 & 29,80 & $\mathrm{~T}$ \\
\hline 698206 & 29,80 & 22,48 & $\mathrm{~T}$ \\
\hline 698207 & 61,03 & 23,63 & $\mathrm{~T}$ \\
\hline PFS 0607 & 52,29 & 23,72 & $\mathrm{~T}$ \\
\hline PFS 0606 & 56,51 & 22,87 & $\mathrm{~T}$ \\
\hline PFS 0605 & 66,35 & 26,28 & $\mathrm{~T}$ \\
\hline PFS 0502 & 70,92 & 33,40 & $\mathrm{~T}$ \\
\hline PFS 0601 & 72,39 & 20,98 & MT \\
\hline
\end{tabular}

${ }^{(1)} \mathrm{T}$, tolerante; S, sensível; MT, moderadamente tolerante; MS, moderadamente sensível. ${ }^{(2)}$ Concentração de $\mathrm{Al}^{3+}$ em $\mathrm{mg} \mathrm{L}^{-1}$. de centeio do norte da Europa, Pinto-Carnide \& Guedes-Pinto (2000) verificaram que a tolerância ao alumínio nos dois grupos foi muito distinta. As populações de centeio português, com grande variabilidade genética, revelaram ser mais tolerantes que as demais cultivares europeias, em concentrações de 30 e $40 \mathrm{mg} \mathrm{L}^{-1} \mathrm{de}^{\mathrm{Al}^{3+}}$. Além disso, essa diferença é explicada pelo fato de ter havido um processo evolutivo de adaptação dessas populações às condições locais de acidez do solo, comuns em Portugal (Pinto-Carnide \& Guedes-Pinto, 2000).

No presente trabalho, não foi constatado nenhum genótipo sensível à concentração de $10 \mathrm{mg} \mathrm{L}^{-1}$ de $\mathrm{Al}^{3+}$, o que corrobora os dados obtidos no trabalho anteriormente citado. Assim sendo, o germoplasma do centeio apresenta excelente potencial de genes para introgressão no trigo ou inclusão no triticale, para se obter tolerância ao alumínio (Pinto-Carnide \& Guedes-Pinto, 2000).

Quanto ao trigo, os genótipos PF 964004 e Toropi apresentaram baixo índice de germinação das sementes, o que inviabilizou a realização da avaliação. No grupo dos tolerantes, foram incluídos os genótipos IPR 85, BRS 194, BRS Angico, BRS Buriti, BRS Guabiju e BRS Guamirim (Tabela 5). O genótipo BRS 209 foi classificado como moderadamente sensível e o CD 104, como sensível, e não diferiram $(\mathrm{p}=0,05)$ do IAC

Tabela 5. Classificação quanto à tolerância e à sensibilidade de genótipos de trigo ao $\mathrm{Al}^{3+}$, definida pelo comprimento da raiz de plantas em cultivo hidropônico em solução de $\mathrm{Al}^{3+}$, que tiveram como controle e testemunha as cultivares de trigo Anahuac 75 (sensível) e IAC 5-Maringá (tolerante).

\begin{tabular}{lccc}
\hline Genótipo & \multicolumn{2}{c}{ Comprimento $(\mathrm{mm})$} & Classificação $^{(1)}$ \\
\cline { 2 - 3 } & $0^{(2)}$ & $2^{(2)}$ & \\
\hline BRS Guamirim & 71,41 & 47,25 & $\mathrm{~T}$ \\
BRS 194 & 47,24 & 31,05 & $\mathrm{~T}$ \\
Jesuíta & 43,83 & 22,83 & $\mathrm{MT}$ \\
CD 104 & 56,11 & 11,46 & $\mathrm{~S}$ \\
IPR 85 & 57,11 & 39,05 & $\mathrm{~T}$ \\
PF 844005 & 31,24 & 12,64 & $\mathrm{MT}$ \\
Anahuac 75 & 61,89 & 13,86 & $\mathrm{~S}$ \\
IAC 5 Maringá & 55,25 & 38,85 & $\mathrm{~T}$ \\
BRS Buriti & 53,97 & 31,35 & $\mathrm{~T}$ \\
IPR 87 & 48,93 & 20,30 & $\mathrm{MT}$ \\
BRS Angico & 60,83 & 32,48 & $\mathrm{~T}$ \\
BRS 229 & 51,55 & 26,14 & $\mathrm{MT}$ \\
BRS Guabiju & 49,43 & 34,88 & $\mathrm{~T}$ \\
BRS 209 & 45,81 & 13,09 & $\mathrm{MS}$ \\
\hline
\end{tabular}

${ }^{(1)}$ T, tolerante; S, sensível; MT, moderadamente tolerante; MS, moderadamente sensível. ${ }^{(2)}$ Concentração de $\mathrm{Al}^{3+} \mathrm{em} \mathrm{mg} \mathrm{L}^{-1}$. 
5-Maringá. Neste caso, $86,7 \%$ da variação observada foi explicada com o modelo ajustado.

Bertan et al. (2006) verificaram, por meio da técnica de hidroponia, diferenças entre 23 genótipos de trigo e variabilidade genética para a tolerância ao alumínio. Isso possibilitou aos autores a identificação dos melhores genótipos para cruzamentos e a obtenção de progênies superiores para tolerância ao alumínio em programas de melhoramento, critério esse empregado por diversos melhoristas (Berzonsky \& Kimber, 1986; Camargo et al., 1998, 2006).

Os genótipos de Ae. tauschii NE 20206-C e NE 20202-A foram incluídos no grupo dos tolerantes e os genótipos NE 20215-Y, RL 5776 e RL 5795 foram incluídos no grupo dos moderadamente sensíveis e os demais genótipos incluídos no grupo do moderadamente tolerantes (Tabela 6). Nessas análises, $81,6 \%$ da variação observada foi explicada com o modelo ajustado.

Para esta espécie, pouquíssimos estudos são relatados. Destaca-se apenas o de Berzonsky \& Kimber (1986), que testaram concentrações de $12 \mathrm{mg} \mathrm{L}^{-1}$ de alumínio em 83 acessos de Ae. tauschii, verificaram que nenhum dos acessos foi tolerante à toxicidade provocada pelo

Tabela 6. Classificação quanto à tolerância e à sensibilidade de acessos de Aegilops tauschii ao $\mathrm{Al}^{3+}$, definida pelo comprimento da raiz de plantas em cultivo hidropônico em solução de $\mathrm{Al}^{3+}$, que tiveram como controle as cultivares de trigo Anahuac 75 (sensível) e IAC 5-Maringá (tolerante).

\begin{tabular}{|c|c|c|c|}
\hline \multirow[t]{2}{*}{ Genótipo } & \multicolumn{2}{|c|}{ Comprimento (mm) } & \multirow[t]{2}{*}{ Classificação $^{(1)}$} \\
\hline & $0^{(2)}$ & $2^{(2)}$ & \\
\hline NE 20238-X & 19,69 & 10,36 & MT \\
\hline NE 20215-Y & 24,52 & 8,59 & MS \\
\hline RL 5786 & 22,47 & 9,15 & MT \\
\hline NE 20234-T & 27,11 & 9,68 & MT \\
\hline NE 20064-A & 21,92 & 8,04 & MT \\
\hline NE 20195-C & 23,74 & 10,29 & MT \\
\hline NE 20206-C & 22,95 & 14,14 & $\mathrm{~T}$ \\
\hline Anahuac 75 & 44,05 & 7,12 & S \\
\hline IAC 5-Maringá & 55,25 & 38,85 & $\mathrm{~T}$ \\
\hline NE 2029-B & 24,19 & 11,16 & MT \\
\hline NE 201196-B & 30,42 & 12,25 & MT \\
\hline NE 201165-Y & 18,35 & 7,44 & MT \\
\hline RL 5776 & 25,63 & 8,77 & MS \\
\hline NE 20207-Y & 25,94 & 10,06 & MT \\
\hline NE 20202-A & 16,22 & 9,65 & $\mathrm{~T}$ \\
\hline NE 20223-A & 28,98 & 13,93 & MT \\
\hline RL 5795 & 27,31 & 8,90 & MS \\
\hline
\end{tabular}

${ }^{(1)}$ T, tolerante; S, sensível; MT, moderadamente tolerante; MS, moderadamente sensível. ${ }^{(2)}$ Concentração de $\mathrm{Al}^{3+}$ em mg L $^{-1}$.
Tabela 7. Classificação quanto à resistência e à sensibilidade dos genótipos de cevada, de triticale, de centeio e de trigo, em condições de campo, em solo sem correção e solo corrigido em $1 / 4$ do índice SMP.

\begin{tabular}{|c|c|c|c|}
\hline Genótipo & Sem correção & Corrigido em $1 / 4$ & Classificação ${ }^{(1)}$ \\
\hline \multicolumn{4}{|c|}{ Cevada } \\
\hline Antártica 04 & 4,0 & 4,0 & $\mathrm{~S}$ \\
\hline MN 698 & 4,0 & 3,5 & MS \\
\hline IPFC 20011 & 4,5 & 3,5 & MS \\
\hline Cevada BR 2 & 3,5 & 3,0 & MS \\
\hline BRS Marciana & 4,5 & 2,5 & MR \\
\hline Alpha & 4,0 & 3,5 & MS \\
\hline BRS 224 & 3,0 & 2,0 & MR \\
\hline FM 404 & 4,0 & 2,5 & $\mathrm{MR}$ \\
\hline Antártica 01 & 4,0 & 3,0 & MS \\
\hline FM 519 & 4,0 & 4,0 & $\mathrm{~S}$ \\
\hline PFC 7802 & 4,0 & 4,0 & $\mathrm{~S}$ \\
\hline Antártica 05 & 4,5 & 4,0 & $\mathrm{~S}$ \\
\hline Cevada BR 1 & 4,0 & 3,0 & MS \\
\hline Harrington & 4,0 & 3,5 & MS \\
\hline \multicolumn{4}{|c|}{ Triticale } \\
\hline PFT 209 & 4,0 & 3,0 & MS \\
\hline BRS 203 & 4,0 & 3,5 & MS \\
\hline BRS Netuno & 4,0 & 3,5 & MS \\
\hline IAPAR 23-Arapoti & 3,0 & 0,5 & AR \\
\hline PFT 0407 & 4,0 & 2,5 & MR \\
\hline PFT 0609 & 3,0 & 2,0 & MR \\
\hline PFT 307 & 4,0 & 2,0 & MR \\
\hline BRS Minotauro & 3,0 & 2,5 & MR \\
\hline PFT 0417 & 5,0 & 3,0 & MS \\
\hline Triticale BR 1 & 3,0 & 4,0 & $\mathrm{~S}$ \\
\hline Embrapa 53 & 3,0 & 1,5 & $\mathrm{R}$ \\
\hline PFT 112 & 4,0 & 1,0 & $\mathrm{R}$ \\
\hline IPR 111 & 4,0 & 1,0 & $\mathrm{R}$ \\
\hline BRS 148 & 3,0 & 3,0 & MS \\
\hline$\underline{B R S \text { Ulisses }}$ & 3,0 & 3,0 & MS \\
\hline \multicolumn{4}{|c|}{ Centeio } \\
\hline 698210 & 4,0 & 2,0 & MR \\
\hline PFS 0504 & 1,0 & 1,0 & $\mathrm{R}$ \\
\hline 698206 & 5,0 & 2,0 & MR \\
\hline 698207 & 4,0 & 2,0 & $\mathrm{MR}$ \\
\hline PFS 0607 & 3,0 & 1,0 & $\mathrm{R}$ \\
\hline PFS 0606 & 2,0 & 0,5 & AR \\
\hline PFS 0605 & 2,0 & 1,0 & $\mathrm{R}$ \\
\hline PFS 0502 & 2,0 & 0,5 & $\mathrm{AR}$ \\
\hline PFS 0601 & 0,5 & 0,5 & AR \\
\hline PFS 0604 & 1,0 & 1,0 & $\mathrm{R}$ \\
\hline PFS 0603 & 2,0 & 1,0 & $\mathrm{R}$ \\
\hline $\mathrm{PF} 0501$ & 2,0 & 1,0 & $\mathrm{R}$ \\
\hline Centeio BR 1 & 2,0 & 0,5 & $\mathrm{AR}$ \\
\hline BRS Serrano & 2,0 & 0,5 & $\mathrm{AR}$ \\
\hline IPR 89 & 3,0 & 3,0 & MS \\
\hline \multicolumn{4}{|c|}{ Trigo } \\
\hline Anahuac 75 & 5,0 & 4,0 & $\mathrm{~S}$ \\
\hline BRS Buriti & 4,0 & 3,0 & MS \\
\hline IPR 87 & 4,5 & 4,0 & $\mathrm{~S}$ \\
\hline BRS Angico & 4,0 & 3,0 & MS \\
\hline BRS 229 & 4,0 & 2,0 & MR \\
\hline BRS Guabiju & 5,0 & 3,0 & MS \\
\hline BRS 209 & 4,5 & 3,0 & MS \\
\hline Toropi & 4,0 & 1,0 & $\mathrm{R}$ \\
\hline BRS Guamirim & 5,0 & 2,0 & MR \\
\hline BRS 194 & 4,5 & 1,0 & $\mathrm{R}$ \\
\hline Jesuíta & 4,5 & 2,0 & MR \\
\hline CD 104 & 5,0 & 4,0 & $\mathrm{~S}$ \\
\hline IAC 5-Maringá & 3,0 & 2,0 & MR \\
\hline IPR 85 & 4,5 & 3,0 & MS \\
\hline PF 844005 & 5,0 & 5,0 & $\mathrm{~S}$ \\
\hline BRS Tarumã & 4,5 & 3,0 & MS \\
\hline
\end{tabular}

(1) $\mathrm{AR}$, altamente resistente $(0,50-0,80)$; $\mathrm{R}$, resistente $(0,81-1,50)$; MR, moderadamente resistente $(1,51-2,50)$; MS, moderadamente suscetível $(2,51-3,50) ; \mathrm{S}$, suscetível $(3,51-4,50) ; \mathrm{AS}$, altamente suscetível $(4,51-5,0)$. 
alumínio, o que provavelmente tenha ocorrido em razão da elevada concentração testada. Ae. tauschii é o doador do genoma "D" de T. aestivum e essa última espécie já apresenta sintomas de sensibilidade a 2 mg L ${ }^{-1}$; concentrações acima desse valor certamente acarretaria maior sensibilidade por parte dos acessos.

Nos experimentos realizados em condições de campo com a cevada, nenhum dos genótipos mostrouse resistente (Tabela 7). Quatro cultivares $(44,4 \%)$ apresentaram resultados semelhantes em campo e em hidroponia: Alpha, BRS 224, FM 404 e Harrington. As outras cinco $(55,6 \%)$ apresentaram resultados contraditórios: em cultivo hidropônico, mostraram ser tolerantes ou moderadamente tolerantes; e, em campo, suscetíveis ou moderadamente suscetíveis. Como no experimento em campo, os genótipos ficam sujeitos a muitas outras variáveis - e não somente à concentração de alumínio - que podem ter influenciado o comportamento desses genótipos, embora essa espécie seja descrita como altamente suscetível ao alumínio (Aniol \& Gustafson, 1984).

Quanto à resposta dos genótipos de triticale nos dois experimentos, oito cultivares $(57,1 \%)$ obtiveram avaliações idênticas em cultivo hidropônico e ensaio a campo: BRS 203, BRS Netuno, IAPAR 23-Arapoti, PFT 307, Triticale BR 1, Embrapa 53, PFT 112 e IPR 111. Outras $(28,6 \%)$ apresentaram padrões semelhantes entre os índices de resistência/tolerância e suscetibilidade/sensibilidade: PFT 209, PFT 0407, PFT 0609 e BRS Minotauro. Somente duas cultivares $(14,3 \%)$ apresentaram avaliações diferentes: BRS Ulisses e BRS 148.

No caso do centeio, tanto no ensaio em campo quanto no cultivo hidropônico, nenhum genótipo mostrou-se suscetível ou sensível ao $\mathrm{Al}^{3+}$. Quatro linhagens $(26,7 \%)$ obtiveram avaliações idênticas: 698206, 698207, PFS 0601 e PFS 0604. Uma cultivar (6,6\%), IPR 89, apresentou resultado diferente. Dez genótipos $(66,7 \%)$ apresentaram padrão semelhante de resistência/tolerância em ambos os experimentos: 698210, PFS 0504, PFS 0607, PFS 0606, PFS 0605, PFS 0502, PFS 0603, PF 0501, Centeio BR 1 e IPR 89.

Quanto aos genótipos de trigo, comparando-se os ensaios em campo e em hidroponia, seis cultivares (42,9\%) apresentaram reações idênticas: Anahuac 75, BRS Buriti, BRS Angico, BRS 229, BRS 194 e CD 104. Cinco $(35,7 \%)$ apresentaram reação semelhante de resistência/tolerância em ambos os experimentos:
IPR 87, BRS 209, BRS Guamirim, IAC 5-Maringá e PF 844005 ; e três cultivares $(21,4 \%)$ apresentaram resultados diferentes: BRS Guabiju, Jesuíta e IPR 85.

Resultados obtidos por Baier et al. (1995) em trigo apresentaram alto coeficiente de correlação entre as avaliações em cultivo hidropônico e em campo $(\mathrm{r}=0,71-0,85 ; \mathrm{p}<0,001)$. Os autores concluíram que a análise hidropônica identifica níveis de tolerância ao alumínio em inúmeros genótipos, o que permite o uso deste método em programas de melhoramento para $\mathrm{o}$ desenvolvimento de cultivares tolerantes ao $\mathrm{Al}^{3+}$.

Ressalta-se ainda que as comparações foram feitas tendo-se relacionado os resultados de comprimento da raiz dos cultivos hidropônicos com as avaliações visuais fenotípicas dos ensaios em campo. Os resultados deste trabalho corroboram os obtidos por Voss et al. (2006), em que o cultivo hidropônico possibilita a realização de estudos genéticos e de caracterização de germoplasma específico para a tolerância ao alumínio.

\section{Conclusão}

1. Os genótipos de triticale, centeio e trigo apresentam elevada similaridade quanto à tolerância ao alumínio em cultivo hidropônico e ensaio em campo.

2. Há relação direta entre a tolerância/sensibilidade e resistência/suscetibilidade ao alumínio dos genótipos de trigo, cevada, triticale e centeio, em cultivo hidropônico e em campo.

3. A seleção inicial de cereais de inverno em cultivo hidropônico é uma ferramenta de apoio eficiente aos programas de melhoramento genético para tolerância ao alumínio.

\section{Agradecimentos}

Aos pesquisadores da Embrapa Trigo, Márcio Voss, pela colaboração, Euclydes Minella, pela escolha dos genótipos de cevada, Márcio Nicolau, pelas análises estatísticas e ao Generation Challenge Programme (CGIAR), pelo auxílio financeiro.

\section{Referências}

ANIOL, A.; GUSTAFSON, J.P. Chromosome location of genes-controlling aluminum tolerance in wheat, rye, and triticale. Canadian Journal of Genetics and Cytology, v.26, p.701-705, 1984.

BAIER, A.C.; SOMERS, D.J.; GUSTAFSON, J.P. Aluminum tolerance in wheat: correlating hydroponic evaluations with field and soil performances. Plant Breeding, v.114, p.291-296, 1995. 
BERTAN, I.; CARVALHO, F.I.F. de; OLIVEIRA, A.C. de; SILVA, J.A.G. da; BENIN, G.; VIEIRA, E.A.; SILVA, G.O. da; HARTWIG, I.; VALÉRIO, I.P.; FINATTO, T. Dissimilaridade genética entre genótipos de trigo avaliados em cultivo hidropônico sob estresse por alumínio. Bragantia, v.65, p.55-63, 2006.

BERZONSKY, W.A.; KIMBER, G. Tolerance of Triticum species to aluminum. Plant Breeding, v.97, p.275-278, 1986.

CAMARGO, C.E. de O.; FELICIO, J.C.; FERREIRA FILHO, A.W.P.; LOBATO, M.T.V. Tolerância de genótipos de trigo comum, trigo duro e triticale à toxicidade de alumínio em soluções nutritivas. Bragantia, v.65, p.43-53, 2006.

CAMARGO, C.E. de O.; FERREIRA FILHO, A.W.P.; FREITAS, J.G. de. Avaliação de genótipos de centeio, triticale, trigo comum e trigo duro quanto à tolerância ao alumínio em solução nutritiva. Scientia Agricola, v.55, p.227-232, 1998.

GUIMARÃES, C.T. Caracterização da variabilidade genética e alélica da tolerância ao alumínio em gramíneas para sua utilização em programas de melhoramento. Sete Lagoas: Embrapa Milho e Sorgo, 2005. 41p.

NARASIMHAMOORTHY, B.; BLANCAFLOR, E.B.; BOUTON, J.H.; PAYTON, M.E.; SLEDGE, M.K. A comparison of hydroponics, soil, and root staining methods for evaluation of aluminum tolerance in Medicago truncatula (barrel Medic) germoplasm. Crop Science, v.47, p.321-328, 2007.
PINTO-CARNIDE, O.; GUEDES-PINTO, H. Differential aluminum tolerance of Portuguese rye populations and North European rye cultivars. Agronomie, v.20, p.93-99, 2000.

SAS INSTITUTE. SAS user's guide. Version 9.1. Cary: SAS Institute, 2004.

SOUSA, C.N.A. de. Classification of Brazilian wheat cultivars for aluminum toxicity in acid soils. Plant Breeding, v.117, p.217-221, 1998.

STRECK, E.V.; KÄMPF, N.; DALMOLIN, R.S.D.; KLAMT, E.; NASCIMENTO, P.C. do; SCHNEIDER, P.; GIASSON, E.; PINTO, L.F.S. Solos do Rio Grande do Sul. Porto Alegre: Emater/RS, 2002. 126p.

VOSS, M.; SOUSA, C.N.A. de; BAIER, A.C.; NASCIMENTO JÚNIOR, A.; BOFF, T. Método de avaliação de tolerância à toxidez de alumínio em trigo, em condições de hidroponia, na Embrapa Trigo. Passo Fundo: Embrapa Trigo, 2006. 16p. (Embrapa Trigo. Documentos online, 67).

WANG, J.-P.; RAMAN, H.; ZHANG, G.-P.; MENDHAM, N.; ZHOU, M.-X. Aluminium tolerance in barley (Hordeum vulgare L.): physiological mechanisms, genetics and screening methods. Journal of Zhejiang University SCIENCE B, v.7, p.769-787, 2006.

Recebido em 17 de outubro de 2009 e aprovado em 29 de janeiro de 2009 